\title{
Age of Onset in Schizophrenia Spectrum Disorders: Complex Interactions between Genetic and Environmental Factors
}

\author{
Laura Mandelli ${ }^{\bowtie}$, Elena Toscano, Stefano Porcelli, Chiara Fabbri, and Alessandro Serretti \\ Department of Biomedical and Neuromotor Sciences, Institute of Psychiatry, University of Bologna, Bologna, Italy
}

\begin{abstract}
In this study we evaluated the role of a candidate gene for major psychosis, Sialyltransferase (ST8SIA2), in the risk to develop a schizophrenia spectrum disorders, taking into account exposure to stressful life events (SLEs). Eight polymorphisms (SNPs) were tested in 94 Schizophreniainpatients and 176 healthy controls. Schizophrenia patients were also evaluated for SLEs in different life periods. None of the SNPs showed association with schizophrenia. Nevertheless, when crossing genetic variants with childhood SLEs, we could observe trends of interaction with age of onset. Though several limitations, our results support a protective role of ST8SIA2 in individuals exposed to moderate childhood stress. Psychiatry Investig 2016;13(2):247-249
\end{abstract}

Key Words Schizophrenia, Age of onset, ST8SIA2, Stressful life events, Gene-environment interaction.

\section{INTRODUCTION}

Schizophrenia spectrum disorders is a clusters of syndromes (schizophrenia, schizoaffective disorder, schizophreniform disorder and not otherwise specified psychotic disorder) characterized by disruptive psychopathology that involves cognition, emotion, perception, and several aspects of behavior. The expression of these disturbances varies among patients and over time, but the effects of illness are severe, often long lasting and worsening in most of the patients. ${ }^{1}$

It is well known that schizophrenia has a high heritability, as observed in family and twin studies ${ }^{2}$ and that genetic factors play an important role in the etiology of the disorder. ${ }^{3}$ Nevertheless, psychosocial influences may play a significant role as well, and the interaction between genetic and environmental risk factors can profoundly influence disease risk, illness features, response to treatments and course of disease over time. ${ }^{4,5}$ In the recent years, a considerable number of studies of geneenvironment interactions $(\mathrm{G} \times \mathrm{E})$ in schizophrenia and other associated disorders have been performed, mainly employing

Received: February 27, 2015 Revised: April 24, 2015

Accepted: May 4, 2015 Available online: January 11, 2016

$\triangle$ Correspondence: Laura Mandelli, $\mathrm{PhD}$

Department of Biomedical and Neuromotor Sciences, Institute of Psychiatry, University of Bologna, Italy, V.le C. Pepoli 5, Bologna 40123, Italy Tel: +39051524100, Fax: +39051521030, E-mail: laura.mandelli@unibo.it

@ This is an Open Access article distributed under the terms of the Creative Commons Attribution Non-Commercial License (http://creativecommons.org/licenses/bync/3.0) which permits unrestricted non-commercial use, distribution, and reproduction in any medium, provided the original work is properly cited. a hypothesis-based genetic approach (i.e., testing candidate genes hypothesized as involved in their pathogenesis). ${ }^{6}$

In our study, we analyzed a relatively novel candidate gene coding for Sialyltransferase (ST8SIA2) that has been implicated in the susceptibility to schizophrenia in previous studies. ${ }^{7.8}$ Sialyltransferase is responsible of the post-translational glycosylation of the neuronal cell adhesion molecule (NCAM). NCAM, in its polysialylated form (PSA-NCAM), plays an important role in processes such as neuronal migration and axon path-finding, synaptogenesis and plasticity. This occurs via masking the adhesive properties of NCAM through steric repulsion, after the addition of large hydrophilic glycopolymers, which facilitates movement through intracellular space for both neurons and neuronal processes. ${ }^{9} 10$ Therefore, Sialyltransferase, by modulating the adhesive properties of NCAM may be critically involved in the pathophysiology of schizophrenia and other neuropsychiatric disorders, ${ }^{11}$ potentially trough $\mathrm{G} \times \mathrm{E}$ interaction. Indeed, in a previous work, we found ST8SIA2 influencing clinical features in major depression only depending on exposure to Stressful life events (SLEs). ${ }^{12}$ To our knowledge ST8SIA2 has never been tested in Schizophrenia along with SLEs, therefore we here investigateda potential impact of ST8SIA2 on Schizophrenia throughout interactions with environmental factors (SLEs).

\section{METHODS}

In the present study, we tested eight single nucleotide poly- 
morphisms (SNPs) within the ST8SIA2 gene in a sample of 94 Schizophrenia patients and 176 healthy controls. Schizophrenia subjects were recruited among consecutively admitted patients our inpatient psychiatric unit, satisfying criteria for a Schizophrenia spectrum disorder as defined above, and aged between $18-80$. Healthy controls were recruited among hospital staff, volunteers and mainly old individuals in the context of a project on cognitive impairment and dementia in the elderly. All healthy controls were accurately screened for the absence of cognitive impairment/dementia and lifetime history of psychiatric disorders. For both patients and healthy controls, severe or unstable medical or neurological disease represented exclusion criteria. Healthy controls were 84 males and 92 females, aged 83.1 \pm 7.1 years; Schizophrenia patients were 67 males and 27 females aged 42.2 \pm 13.1 . In Schizophrenia subjects, a number of socio-demographic and clinical variables including family history for psychiatric disease, age of onset, substance abuse, suicide attempt and number of suicide attempts, numbers of hospitalizations and current symptomatology by the Positive and Negative Syndrome Scale (PANSS) ${ }^{12}$ were assessed. To account for the environmental influence, in Schizophrenia patients we assessed a number of stressful life events (SLEs) occurring in three different life periods: in childhood, at illness onset and in the past month before current hospitalization by an interview designed to collect adverse events or events requiring effort of adaptation (material available on request).

The following SNPs within ST8SIA2 were evaluated in both Schizophrenia patients and healthy controls by PCRbased techniques (details on request): rs3759917, rs11632521, rs3784722, rs4777989, rs2290492, rs8035760, rs11853992, rs17522085. Hardy-Weinberg Equilibrium (HWE) and Linkage disequilibrium (LD) were tested by the Haploview (software).$^{13}$ Linear analysis were performed by appropriate parametric and non-parametric tests. Linear regression was employed to evaluate the interaction between genetic variants and SLEs on clinical variables collected in the Schizophrenia sample. Haplotype analysis was performed by the R software (http://www.r-project.org/). Considering a significance level of 0.05 , we had a sufficient power (0.80) to detect small-medium effect of $\mathrm{w}=0.17$ in simple case-control analysis.

\section{RESULTS}

Schizophrenia patients were mainly diagnosed for Schizophrenia $(77.8 \%)$ and more than one-third had a positive family history for psychiatric disorders (36.1\%). Mean age of onset was 24.9 \pm 9.4 years and mean total score at the PANSS was 75.4 \pm 21.3 . By allelic analysis, none of the SNPs showed association with Schizophrenia, though the minor A-allele in rs4777989 was slightly over-represented in Schizophrenia patients as compared to controls $(\mathrm{p}=0.08)$. No association could be detected with other variables, including SLEs at different time points. Nevertheless, when crossing genetic variants with SLEs, we could observe a trend of interaction between childhood SLEs, rs2290492 and rs11853992 alleles on disease onset. rs2290492-C allele carriers and rs11853992-A allele carriers showed an older age at disease onset only if they had been exposed to a moderate number of SLEs during childhood (1 or 2), whilst they have an earlier onset if both exposed to no or more than 3 SLEs (Table 1). Though in full linkage disequilibrium, we performed haplotype analysis, but none of the SNPs showed association the with analyzed variables, i.e. diagnosis, marital status, psychiatric family history, substance abuse, suicide attempts.

\section{DISCUSSION}

According to these results, variants in ST8SIA2 seem to favor a later development of disease in individuals moderately exposed to childhood stress only. Although the critical role of stress in the early onset of schizophrenia has been recognized, ${ }^{14}$ the exposure to a moderate level of stress in early life may be protective in some individuals. This is an unexpected result because most of the works in literature support a negative role of SLEs on onset, severity and prognosis of psychiatric diseases. However, the large part of the studies focus on exposure to SLEs measured in a specific life period. In this study, we adopted a more comprehensive approach, considering SLEs in childhood, at illness onset and in the past month before current hospitalization. ST8SIA2 modulates the adhesive properties of NCAM which plays an important role in synaptogenesis and plasticity. According to our data, we may hypothesize that in the early development, a moderate stress may promote the activity of NCAM trough the activity of ST8SIA2. This "activation" would not happen in the absence of a sufficient amount of stress, in individuals carriers of ge-

Table 1. rs2290492 and rs11853992 (ST8SIA2) alleles, childhood stressful life events (SLEs) and their interaction on age of onset in Schizophrenic spectrum disorders

\begin{tabular}{|c|c|c|c|}
\hline & B & $\mathrm{F}$ & $\mathrm{p}$ \\
\hline rs2290492 & 0.07 & 0.41 & 0.53 \\
\hline Childhood SLEs & -0.13 & 0.97 & 0.39 \\
\hline rs2290492×Childhood SLEs & 0.30 & 3.06 & 0.06 \\
\hline rs11853992 & 0.07 & 0.41 & 0.53 \\
\hline Childhood SLEs & -0.13 & 0.97 & 0.39 \\
\hline rs11853992×Childhood SLEs & 0.30 & 3.06 & 0.06 \\
\hline
\end{tabular}

Coefficients and results are the same probably because the SNPs were in full Linkage disequilibrium in our sample 
netic differences in ST8SIA2, and it would be ineffective in case of excessive exposure to stress. According to our results, we may hypothesize that stress-induced activation of NCAM during development may favor neuroplastic processes which may eventually protect predisposed individuals against early illness development. Following this hypothesis, NCAM, ST8SIA2 and other factors involved in neuronal development and neuroplasticity may represent the biological basis of resilience to moderate stress. Resilience is a dynamic process that encompasses the attainment of positive adaptation within the context of exposure to adversity that typically exerts major assaults on biological and psychological development. ${ }^{15}$ It is a positive adaptation and maintenance of competent functioning in the face of stress. ${ }^{14}$ Resilient functioning is among the most intriguing and adaptive phenomena of human development.

According to our data, we may hypothesize that genes involved in neuroplasticity, favoring certain individuals in the face of moderated early adversity, may delay or prevent disease manifestation. However, the results obtained, as well as the hypotheses we made, should be taken with caution and just as a suggestion for further investigation. The present study is indeed characterized by several limitations which make the overall conclusions merely speculative. A major limitation of the study is represented by the small sample size, leading to a high risk for positive and negative false findings, and to limited statistical power for multiple testing. We did not applied a correction for multiple testing in order to detect minor effects; on the other hand, this limits the reliability of findings which should be taken as preliminary only. Other interactive effects of potential interest or confounding variables could not be tested in a such a small sample. The small sample size allowed allelic analysis only and we were not able to test dominant/heterozygous effects. Furthermore, in many cases minor alleles had a low frequency and were underrepresented in some groups. SLEs were retrospectively evaluated, with the risk of recall bias and missed information. Further, SLEs were not evaluated in healthy subjects making impossible to test potential differences between cases and controls. Finally a number of variables potentially impacting illness onset other than SLEs were not taken into account in the present study.

In conclusion, genetic variations within ST8SIA2 may modulate age of illness onset depending on exposure to stress in childhood and may potentially favor some individuals against the detrimental effect of stress in liability for psychiatric disease. Further studies on larger and more powered sam- ples are required to investigate the role of neuroplastic factors in mediating the effects of environmental stress in mental health.

\section{REFERENCES}

1. Sadock BJ, Sadock VA. Concise Textbook of Clinical Psychiatry. Philadelphia, PA: Lippicott \& Wilkins; 2008.

2. Lang UE, Puls I, Muller DJ, Strutz-Seebohm N, Gallinat J. Molecular mechanisms of schizophrenia. Cell Physiol Biochem 2007;20:687-702.

3. Roussos P, Haroutunian V. Schizophrenia: susceptibility genes and oligodendroglial and myelin related abnormalities. Front Cell Neurosci 2014;8:5.

4. Iyegbe C, Campbell D, Butler A, Ajnakina O, Sham P. The emerging molecular architecture of schizophrenia, polygenic risk scores and the clinical implications for GxE research. Soc Psychiatry Psychiatr Epidemiol 2014;49:169-182.

5. Tienari P, Wynne LC, Laksy K, Moring J, Nieminen P, Sorri A, et al. Genetic boundaries of the schizophrenia spectrum: evidence from the Finnish Adoptive Family Study of Schizophrenia. Am J Psychiatry 2003;160:1587-1594.

6. Modinos G, Iyegbe C, Prata D, Rivera M, Kempton MJ, Valmaggia LR, et al. Molecular genetic gene-environment studies using candidate genes in schizophrenia: a systematic review. Schizophr Res 2013;150:356-365.

7. Arai M, Yamada K, Toyota T, Obata N, Haga S, Yoshida Y, et al. Association between polymorphisms in the promoter region of the sialyltransferase 8B (SIAT8B) gene and schizophrenia. Biol Psychiatry 2006;59: 652-659.

8. Tao R, Li C, Zheng Y, Qin W, Zhang J, Li X, et al. Positive association between SIAT8B and schizophrenia in the Chinese Han population. Schizophr Res 2007;90:108-114.

9. McAuley EZ, Scimone A, Tiwari Y, Agahi G, Mowry BJ, Holliday EG, et al. Identification of sialyltransferase $8 \mathrm{~B}$ as a generalized susceptibility gene for psychotic and mood disorders on chromosome 15q25-26. PLoS One 2012; 7:e38172.

10. Atz ME, Rollins B, Vawter MP. NCAM1 association study of bipolar disorder and schizophrenia: polymorphisms and alternatively spliced isoforms lead to similarities and differences. Psychiatr Genet 2007;17: 55-67.

11. Hildebrandt H, Muhlenhoff M, Oltmann-Norden I, Rockle I, Burkhardt H, Weinhold B, et al. Imbalance of neural cell adhesion molecule and polysialyltransferase alleles causes defective brain connectivity. Brain 2009;132:2831-2838.

12. Mandelli L, Emiliani R, Porcelli S, Fabbri C, Albani D, Serretti A. Genes involved in Neuroplasticity and Stressful Life Events act on the shortterm response to antidepressant treatment: a complex interplay beetween genetics and environment. Hum Psychopharmacol 2014;29:338-391.

13. Kay SR, Fiszbein A, Opler LA. The positive and negative syndrome scale (PANSS) for schizophrenia. Schizophr Bull 1987;13:261-276.

14. Barrett JC, Fry B, Maller J, Daly MJ. Haploview: analysis and visualization of LD and haplotype maps. Bioinformatics 2005;21:263-265.

15. Mihali A, Subramani S, Kaunitz G, Rayport S, Gaisler-Salomon I. Modeling resilience to schizophrenia in genetically modified mice: a novel approach to drug discovery. Expert Rev Neurother 2012;12:785-799.

16. Cicchetti D, Rogosch FA, Thibodeau EL. The effects of child maltreatment on early signs of antisocial behavior: genetic moderation by tryptophan hydroxylase, serotonin transporter, and monoamine oxidase A genes. Dev Psychopathol 2012;24:907-928. 